\title{
Noise induced creation and annihilation of solitons in dispersion managed fiber oscillators
}

\section{Tesfay G. Teamir, F. Ömer Ilday}

Tesfay G. Teamir, F. Ömer Ilday, "Noise induced creation and annihilation of solitons in dispersion managed fiber oscillators," Proc. SPIE 10254, XXI International Symposium on High Power Laser Systems and Applications 2016, 1025409 (13 January 2017); doi: 10.1117/12.2256324

SDIE Event: XXI International Symposium on High Power Laser Systems and Applications, 2016, Gmunden, Austria 


\section{Noise Induced Creation and Annihilation of Solitons in Dispersion Managed Fiber Oscillators \\ Tesfay G. Teamir ${ }^{1, *}$ and F. Ömer Ilday ${ }^{1,2}$ \\ 1. Department of Physics, Bilkent University, Ankara, 06800, Turkey \\ 2. Department of Electrical and Electronics Engineering, Bilkent University, Ankara, 06800, Turkey \\ *teamir@fen.bilkent.edu.tr}

\section{Introduction}

Optical solitons and their interaction with other solitons or with dispersive wave shed by solitons under modulation instabilities or perturbation constitute a versatile experimental and theoretical platform for studying the nature of complex dynamics occurring in laser cavities [1-3] in addition to common physical principles in terms with a range of other nonlinear, non-equilibrium, coupled systems outside of optics.

A soliton is energy localization of dissipative structures of electric field which evolves from noise in laser cavities. It is stationary solution of nonlinear Schrödinger equation that balances the effects of chromatic dispersion with nonlinearity during propagation in a medium. Strong pumping in soliton regime drives a laser system in to a multi pulsing self-organized system. Such a system in fiber medium is ubiquitous and always attracts research interest.

Multisoliton pulses or soliton bunches generated from different systems through a short and long range interaction due to acoustic waves generated from electrostriction and its perturbation induced refractive index change of the medium by a propagating pulse on the next pulse in the neighborhood [4]. A short range interaction can occur as a result of pulses overlapping, acoustoptic interaction or it can occur when dispersive waves at the tail of pulses interact with a back ground field or with solitons near to its $[1,4,5]$.

\section{Experimental results}

The experimental was conducted as schematically described in [6]. It is a fiber laser mode locked by NPE with physical parameters of total length of $\sim 350 \mathrm{~cm}$ passive fiber, $27 \mathrm{~cm}$ of Yb-doped gain segment with net GVD of $8000 \mathrm{fs}^{2}$ and repetition rate of $44.2 \mathrm{MHz}$. By scanning the pump power, the oscillator can exhibit a range of dynamics that vary from a single stable DM soliton state to a multi soliton bound states with fixed and varying phase as well as temporal separation. As we scan the pump power soliton states with constantly energy exchanging solitons, varying or vibrating temporal separation which often are followed by transformation from one to another random solitonic bound states. This critical behavior is caused by long and short soliton-soliton, soliton-generated dispersive wave or optomechanical interaction. The RIN of these states is found to be higher than stable soliton states and is often times characterized by acoustic peak on the RIN spectrum. Further increment of pump power creates a random new soliton state with smaller (Fig 1(I) (a) energy level indicated by (g)) or larger (Fig 1(I) (a) energy level indicated by(h)) number of solitons that cannot be justified only with quantization of soliton energy.
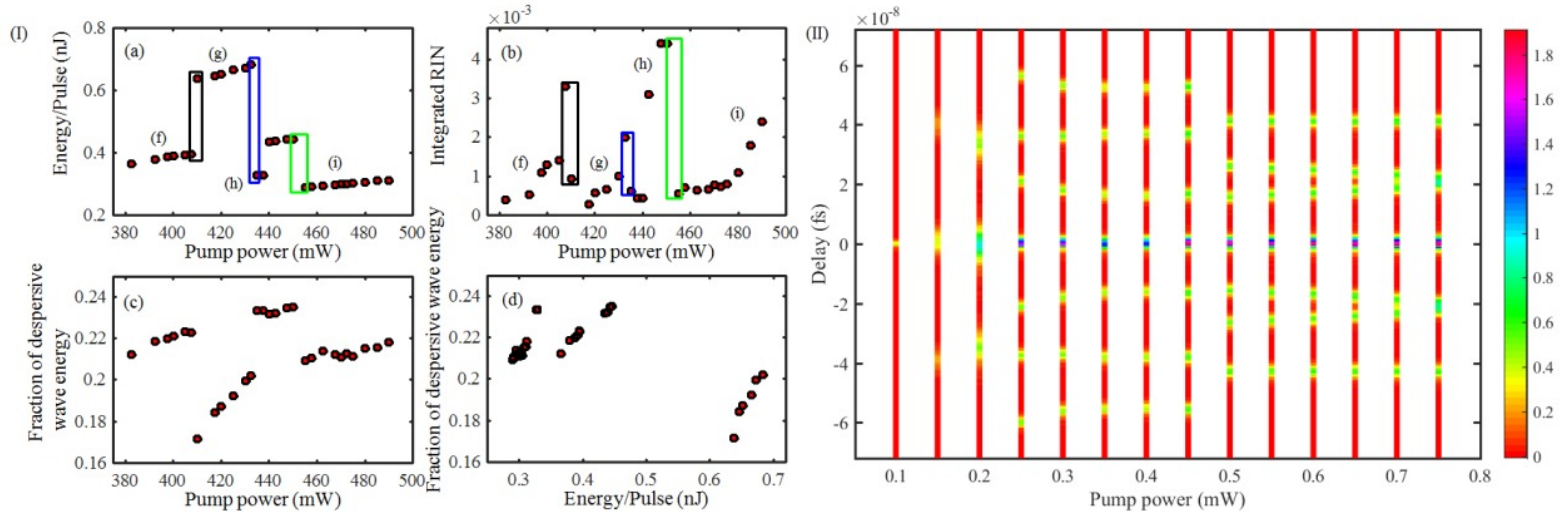

Fig. 1: (I) Shows relationship between energy/pulse, Integrated RIN and fraction of energy going to dispersive waves as pump power is scanned (Note: the colored boxes are just to highlight transition points). (II) Top view showing autocorrelation function of soliton states, its interaction and its transition to other states as the pump power is scanned.

Experimental comparison of formation and disappearance mechanism of DM solitons inside punch of soliton states is done while the oscillator is operating at its fundamental repetition rate. Irrespective of the level of pump power it is formed, the noise level of every solitonic state shows similar dependence on pump power as shown in Fig. 1(II) (b). Energy per pulse and the relative intensity of generated dispersive wave also show similar behaviour at a given 
state before a state transformation takes place. The transition mechanism involves interaction of solitons through exchange of energy, Acoustic wave generated by electrostriction induced interaction [1], bunching of solitons through forward Brillouin scattering [3], effective attractive and repulsive force (as shown in Fig 1 (II)) which resulted from the higher order dispersion and nonlinearity perturbations [7, 8]. Every transition points are characterized by relatively highest RIN noise. The interaction dynamics of these solitons in side cluster exhibits in a similar way to interaction of active matter and should be governed by self-organization far from equilibrium Thermostatistical systems. These results give important insight in characterizing the oscillator dynamics regimes, in helping exploration of a new dynamics and should be a research platform for studding far from equilibrium processes as well.

Dispersive waves are generated by perturbation that a soliton experiences due to discrete nature of the effects of dispersion, nonlinearity and loss/gain as it propagates in the oscillator. The fraction of dispersive wave is calculated as the energy distributed over the wing of pulse spectrum divided by the total pulse energy distributed over the spectrum. Fig. 2 indicates the effect of pulse interaction with dispersive wave. The pump power is the same but the pulse has slightly different duration ( $255 \mathrm{fs}$ and $212 \mathrm{fs}$ ) and approximated energy difference of $5 \mathrm{pJ}$ with the shortest being stronger. When the relative intensity of Kelly side bands is stronger (fig. 2(b)), which is a manifestation of strong coupling between soliton and dispersive wave, the soliton becomes unstable as also theoretically predicted in ref. $[9,10]$. Hence integrated relative intensity noise of the system which can be seen from fig. 2(d) increases by fivefold. Asymmetry on the side bands is indication of relative cavity loses on the longer and shorter wavelength part of the pulse. This change in asymmetry together with energy difference between these coexisting soliton states is a manifestation of different nonlinear polarization evolution in the cavity for the two cases. These to lasing states can be equivalently driven from each other either by increasing or decreasing pump power.
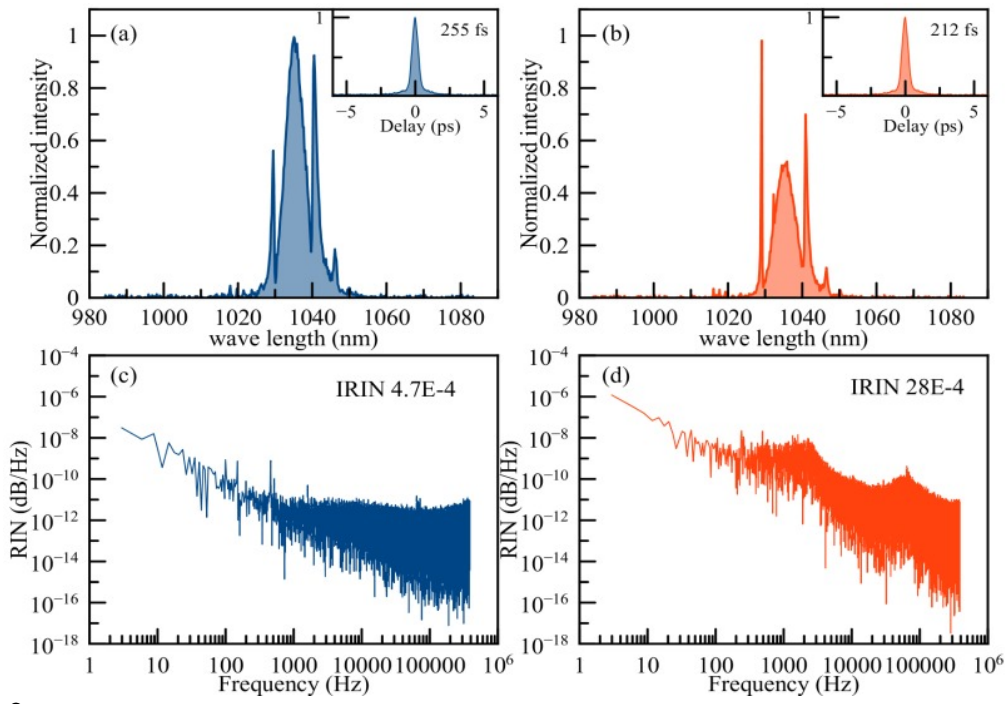

Fig. 2: (I) Shows wavelength ((a) and (b)) Autocorrelation function (inset of ((a) and (b))) and its corresponding noise spectrum ((c) and (d)) for a stable pulse (blue) and for a stronger pulse interacting with dispersive wave (red) at the same pump power of $360 \mathrm{~mW}$.

The spatiotemporal dynamics of this two states is shown in fig 3 below. It shows the intensity distribution of pulse power along the cavity taken in $35 \mathrm{~s}$ with $12 \mathrm{Ghz}$ photodiode and a $2.5 \mathrm{GHz}$ oscilloscope. The laser was operating at its third harmonics with a repetition rate of $148.4 \mathrm{MHz}$. There are three pulses per cavity round trip. In the first dynamics the three pulses are moving at a fixed position relative to each other. But in the second dynamics these pulses can't maintain their relative temporal position due to the interaction between each other initiated by relatively intense generated dispersive waves. As the intensity of side bands gets stronger the pulses in the cavity experience alternating effective attractive and repulsive forces between each other as can be seen from magnified portion of fig. 3(b). As the energy going to dispersive wave or to the soliton side band the temporal position of solitons in the cavity start to jitter as shown in fig $2 \mathrm{~b}$. This can be fundamentally limiting quality of information especially in telecommunication and information processing application. Unlike the results of ref. [11], the RIN is associated with the intensity of side band rather than the operating harmonics of the repetition rate. 

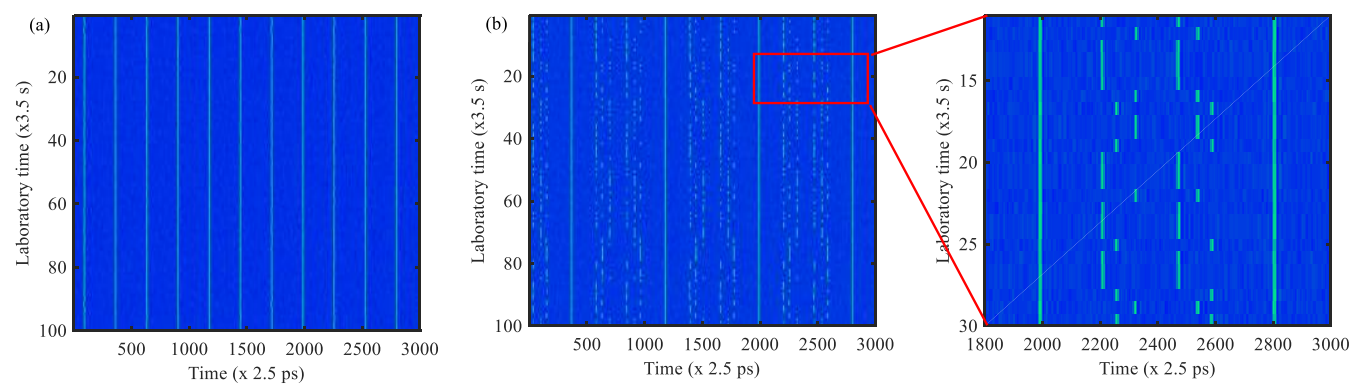

Fig. 3: (I) Spatiotemporal dynamics of the two states indicated in fig. 2(a) and 2(b) respectively which indicates the dependence of intensity distribution of power and its RIN noise in the cavity.

Such events can also occur when one scans the pump power inside the cavity. We scan the pump power from 175 $\mathrm{mW}$ to $230 \mathrm{~mW}$ pump power. We explore and examine the effects of pump power on the interaction of dispersive wave and single and double pulsing regimes whose autocorrelation trace, optical spectrum and RIN information indicated in Fig. 4. Mode locking starts $160 \mathrm{~mW}$ with relatively higher integrated RIN level (0.032 at $173 \mathrm{~mW})$. At $176 \mathrm{~mW}$ the integrated RIN is 0.0025 . As the pump power increases integrated RIN decreases up to $210 \mathrm{~mW}$ (2e-4) and it increases afterwards till it breaks up in to two solitons at $228 \mathrm{~mW}$. In our case spectral side bands are related with stability of laser or soliton states. The bound soliton states are more stable when the intensity of the spectral side bands are relatively smaller as can be seen also from Fig. $b(b)$. The relative intensity of spectral sidebands increases and their relative position from the central frequency decreases. Effective attractive and repulsive forces are associated with it. As we have seen for ANDi laser also the integrated RIN is higher around the mode locking threshold. Hence, Relatively larger value of the RIN at lower pump power (Fig. 4(c)) is related with the closeness of the pump power to the threshold pump power to mode locking.

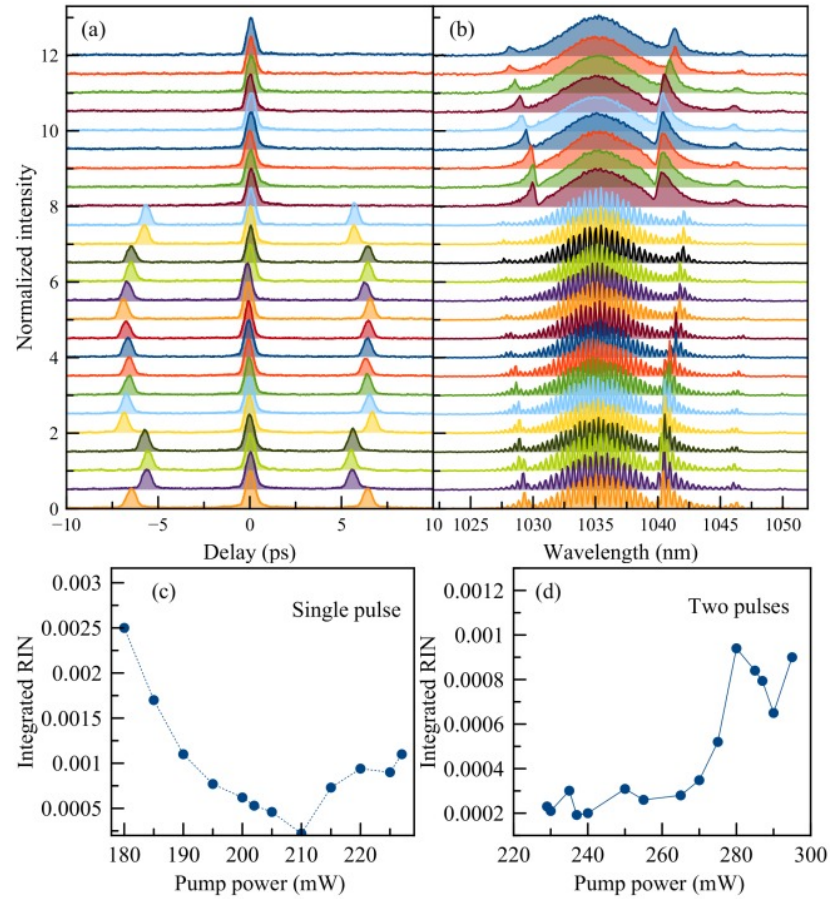

Fig 4: (a) autocorrelation function (b) corresponding spectrum (c) and (d) Integrated RIN Dependence on pump power for the two soliton states namely single and double pulsing states respectively. 


\section{Simulation results}

Simulation based on solution of nonlinear Schrödinger equation is performed to examine the effect of dispersive wave and soliton pulse interaction on the oscillator stability by scanning intracavity pump power. As the pump power increases both the dispersive wave energy and soliton energy increases. The separation between the central frequency of the pulse and the side band decreases with increasing pump power as shown in Fig. 5(a). As the side band gets stronger it starts to interact with the soliton through energy competition, hence the optical spectrum of the pulse start to oscillate periodically between two shapes as seen in Fig. 5(b). The pulse energy also starts to oscillate at pump power of $110 \mathrm{~mW}$ as indicated on Fig. 5(c). The intensity distribution per round trip of the optical spectrum at each pump power is also shown on Fig. 5(d-g). As the intensity of dispersive wave increases interaction with the main soliton is intensified and finally destabilizes the oscillator output, hence intensity noise increases. This result is in a good agreement with our experiment and theories in literature.
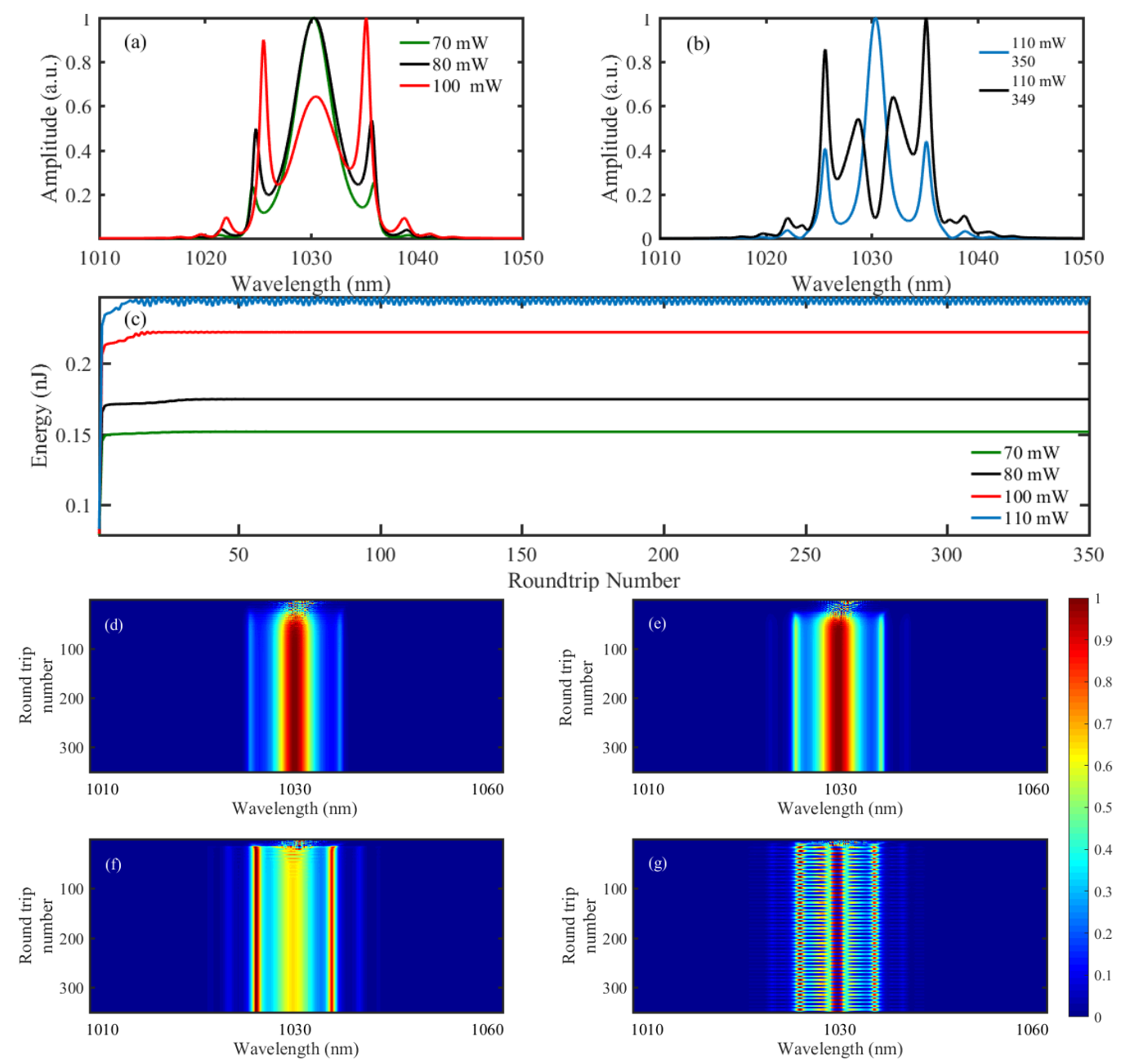

Fig 5: simulation result showing evolution of optical pulse spectrum at the end of 350 cavity round trip for indicated level of pumping power (a) and (b). (c) Pulse energy evolution in the cavity at different pumping powers. (d) - (g) optical Spectral evolution dynamics per cavity round trip for corresponding pump powers indicated on (c).

\section{Conclusion}

We discussed the experimental formation and disappearance mechanism of DM solitons inside fiber oscillator. Irrespective of the level of pump power it is formed, the noise level of every solitonic state showed similar dependence on pump power, energy per pulse and the relative intensity of generated dispersive wave before a state 
transformation takes place. Every transition points are characterized by relatively highest noise level which is due to long range soliton soliton and short range soliton-dispersive wave interaction. This elevated noise dictates irreversible creation or annihilation of DM solitons in the oscillator.

\section{References}

3. J. K. Jang, Miro Erkintalo, S. G. Murdoch and S. Coen, "Ultraweak long-range interactions of solitons observed over astronomical distances," Nat. Photon. 7, 657-663 (2013)

4. C. Rotschild, B. Alfassi, O. Cohen' and M. Segev, "Long-range interactions between optical solitons," Nature Physics 2, 769 - 774 (2006)

5. M. Erkintalo, K. Luo, J. K. Jang, S. Coen, S. G. Murdoch, "Bunching of temporal cavity solitons via forward Brillouin scattering," New J. Phys, 17 (2015).

6. M. Pang, W. He, X. Jiang, and P. St. J. Russell, "All-optical bit storage in a fibre laser by optomechanically bound states of solitons ,” Nat. Photonics 10, 454-458 (2016).

7. D. Y. Tang et al., "Soliton interaction in a fiber ring laser", Phys. Rev. E 72, 016616 (2005).

8. T. Teamir, P. Elahi, L. Budunoglu, K. Gurel, and F. Ilday, "Influence of pump noise on mode-locked fiber oscillators," Advanced Solid State Lasers, ATh2A.34 (2015).

9. A. Hause, F. Mitschke, "Higher-order equilibria of temporal soliton molecules in dispersion-managed fibers," Phys Rev. A 88, 43-53 (2013).

10. A. Hause, F. Mitschke, "Interaction of an optical soliton with a dispersive wave," PRL 95, 213902 (2005).

11. S. M. Kelly, "Characteristic sideband instability of periodically amplified average soliton", Electron. Lett. 28 (8), 806 (1992).

12. J. P. Gordon, "Dispersive perturbations of solitons of the nonlinear Schrödinger equation”, J. Opt. Soc. Am. B 9 (1), 91 (1992).

13. D. Y. Tang, L. M. Zhao, X. Wu, and H. Zhang, "Soliton modulation instability in fiber lasers", Phys Rev. A 80, 023806 (2009). 